\title{
CONSTRUCTING QUATERNIONIC FIELDS
}

\author{
by THERESA P. VAUGHAN
}

(Received 31 July, 1990)

Introduction. Let $K$ be a field of characteristic different from 2, and $a, b$ quadratically independent elements of $K$. Put $J=K(\sqrt{a}, \sqrt{b})$. In [4], Jensen and Yui discuss the question of quaternionic $\left(Q_{8}\right)$ extensions of $J$, and give a survey of known results. In [8], Ware discusses (among other things) some general conditions for, and relations between, the existence of $Q_{8}$ and $D_{4}$ (dihedral) extensions of $K$. A general theorem of Witt [9] says that $J$ will have a quaternionic extension $J(\sqrt{u})$ if and only if there exists a $3 \times 3$ matrix $P$ over $K$ such that $P P^{t}=\operatorname{diag}(a, b, 1 / a b)$, and an appropriate value for $u$ is given in terms of the entries of $P$. The problem of actually finding $P$ in a particular case is not trivial.

Jensen and Yui give an explicit construction for a suitable $P$ in case -1 is a sum of two squares in $K$, and in [3], Cohn gives a suitable $P$ for the case when $J=Q(\sqrt{2}, \sqrt{q})$ with $q$ a prime, $q \equiv 1$ or $3(\bmod 8)$. There are other characterizations of the problem also. The conditions given by Reichardt [5] consist of the solvability of a set of three simultaneous quadratic equations; Bucht [2] gives conditions which amount to the solution of three simultaneous quartic equations.

The original motivation for this paper, was the construction of quaternionic fields over $Q$. To this end, we study in some detail the properties which the field $J$ and the defining element $u$ must have in order that $J(\sqrt{u})$ shall be a $Q_{8}$ extension of $K$. In Section 1 , we give some preliminaries, and characterize non-cyclic normal (over $K$ ) extensions of the form $J(\sqrt{u})$, according to which, and how many of the products $u \sigma(u)$ are square in a quadratic subfield (where $\sigma$ is a $K$-automorphism of $J$ ).

In Section 2, we define a $Q_{8}$ extension $L$ of $J$ to be of Type I if $J$ also admits a dihedral $\left(D_{4}\right)$ extension (and equivalently, a $Z_{2} \times Z_{4}$ extension), and of Type II otherwise. We show that $L$ is of Type I if and only if there exist certain elements $x$ and $y$ in two of the quadratic subfields of $J$, such that $J(\sqrt{u})=J(\sqrt{x y})$. In order to construct a Type I extension, it suffices to construct the matching $D_{4}$ extension and $Z_{2} \times Z_{4}$ extension.

In Section 3, we give a characterization of $Q_{8}$ extensions of $J=Q(\sqrt{n}, \sqrt{m})$ in terms of the norms of algebraic integers in the quadratic subfields of $J$. In Section 4, we construct several types of quaternionic extensions of $Q$, and also give a set of sufficient conditions for a $Q_{8}$ extension which yields a straightforward construction.

I would like to thank the referees for Corollary 2.6, for the reference [8], and for very helpful suggestions in general.

1. Notation and preliminaries. The notation established in this section will be used throughout this paper.

Let $K$ be a field of characteristic different from 2, and let $K^{2}$ denote the set of squares in $K$. Suppose that $K$ contains elements $a_{1}$ and $a_{2}$ such that none of $a_{1}, a_{2}, a_{1} a_{2}$ is a square in $K$. Put $a_{3}=a_{1} a_{2}$. Then $J=K\left(\sqrt{a_{1}}, \sqrt{a_{2}}\right)$ has three quadratic subfields, $K\left(\sqrt{a_{i}}\right)$, $1 \leq i \leq 3$, and the Galois group $\operatorname{Gal}(J / K)$ is $Z_{2} \times Z_{2}$. The three non-trivial $K$ - 
automorphisms are determined by

$$
\sigma_{i}\left(\sqrt{a_{i}}\right)=\sqrt{a_{i}}, \quad \sigma_{j}\left(\sqrt{a_{i}}\right)=-{\sqrt{a_{i}}} \text { for } i \neq j \quad(1 \leq i, j \leq 3) .
$$

Let $u \in J-J^{2}$, and $L=J(\sqrt{u})$. Then $L$ is normal over $K$ if and only if $u \sigma_{i}(u)$ is in $J^{2}$ for all $i=1,2,3$, and then $\operatorname{Gal}(L / K)$ must be a non-cyclic group of order 8 , that is, one of $Z_{2} \times Z_{2} \times Z_{2}, Z_{4} \times Z_{2}, D_{4}$, or $Q_{8}$. In what follows, we assume that $K$ has the properties described above, and $L=J(\sqrt{u})$ is normal over $K$. (In particular, $K$ is not finite, and $K$ has non-cyclic normal extensions of degree 8 over $K$.)

We will use without comment the well-known fact that if $F$ is any field, and $x$ and $y$ are in $F$, then $F(\sqrt{x})=F(\sqrt{y})$ if and only if $x y \in F^{2}$. Also, the following formal identities will be useful.

Lemma 1.1. (a) Suppose that $x^{2}-n y^{2}=t^{2}$. Then

$$
2(x-t)(x+y \sqrt{n})=(x-t+y \sqrt{n})^{2} .
$$

(b) Suppose that $x^{2}-n y^{2}=k t^{2}$. Then

$$
\{\sqrt{x+y \sqrt{n}}+\sqrt{x-y \sqrt{n}}\}^{2}=2 x+2 t \sqrt{k}
$$

Proof. Verify.

COROLlaRY 1.2. If $\alpha=x+y \sqrt{a} \in K(\sqrt{a})$, and if $N_{K}(\alpha)=x^{2}-a y^{2}=t^{2}$ for some $t \in K$, then $K(\sqrt{a}, \sqrt{\alpha})=K(\sqrt{a}, \sqrt{b})$ for some $b \in K$.

Since $u \sigma_{i}(u) \in J^{2}(i=1,2,3)$, and also $u \sigma_{i}(u) \in K\left(\sqrt{a_{i}}\right)$, then for each $i=1,2,3$, one of the following statements must be true:

$$
\begin{gathered}
u \sigma_{i}(u) \in\left(K\left(\sqrt{a_{i}}\right)\right)^{2} \\
u \sigma_{i}(u) \in a_{j}\left(K\left(\sqrt{a_{i}}\right)\right)^{2} \text { for } i \neq j \quad(1 \leq j \leq 3) .
\end{gathered}
$$

The next result follows almost immediately from this observation.

LEMMA 1.3. If $L=J(\sqrt{u})$ is normal over $K$, then there exist $x_{i}=K\left(\sqrt{a_{i}}\right)(i=1,2,3)$ such that $L=J\left(\sqrt{x_{1} x_{2} x_{3}}\right)$.

Proof. Since for each $i=1,2,3$ either $\left({ }^{*}\right)$ or $\left({ }^{* *}\right)$ must be true, then $N_{K}(u)$ must be a square in $K$. Write $u \sigma_{i}(u)=m_{i} y_{i}^{2}$, where $m_{i} \in\left\{1, a_{1}, a_{2}, a_{3}\right\}$ and $y_{i} \in K\left(\sqrt{a_{i}}\right),(i=1,2,3)$. Then we have $u^{2} N_{K}(u)=m_{1} m_{2} m_{3}\left(y_{1} y_{2} y_{3}\right)^{2}$. The quantity $m_{1} m_{2} m_{3}$ must be square in at least one of the fields $K\left(\sqrt{a_{i}}\right)$; suppose for example that $m_{1} m_{2} m_{3}=b^{2}$ for some $b \in K\left(\sqrt{a_{1}}\right)$. Then $u= \pm\left(b y_{1}\right) y_{2} y_{3}$, and we can take $x_{1}= \pm b y_{1}, x_{2}=y_{2}$, and $x_{3}=y_{3}$.

We shall see that the truth-values of the statements $\left({ }^{*}\right)$ and $\left({ }^{* *}\right)$ determine the field lattice of $L$ over $K$. First we need a definition.

Definition 1.4. Let $u \in J-J^{2}$, and assume the notation given above. The ordered triple $S(u)=\left(s_{1}, s_{2}, s_{3}\right)$ is defined by: $s_{i}=0$ if $u \sigma_{i}(u) \in\left(K\left(\sqrt{a_{i}}\right)\right)^{2}$, and $s_{i}=1$ if $u \sigma_{i}(u) \epsilon$ $a_{j}\left(K\left(\sqrt{a_{i}}\right)\right)^{2}$ for some $i \neq j, 1 \leq j \leq 3$. If a fuller notation is needed, we will write $s_{i}=s_{i}(u)$.

TheOREM 1.5. Assume all the notation given above. For each $i, 1 \leq i \leq 3, s_{i}=0$ if and only if there exists an element $t \in K\left(\sqrt{a_{i}}\right)$ such that $t u \in J^{2}$, that is, if and only if $J(\sqrt{u})=J(\sqrt{t})$ 
Proof. Fix $i, 1 \leq i \leq 3$, and put $s_{i}=s, \sigma_{i}=\sigma$, and $a_{i}=a$. Choose $j \neq i$, and put $b=a_{j}$. Since $u \in J$, we can write $u=x+y \sqrt{b}$, where $x, y \in K(\sqrt{a})$. Suppose first that $s=0$; then there is some $z \in K(\sqrt{a})$ such that

$$
u \sigma(u)=x^{2}-b y^{2}=z^{2}
$$

and then, from the identity (ID1), we have

$$
2(x-z) u=(x-z+y \sqrt{b})^{2} \in J^{2} .
$$

Then $J(\sqrt{u})=J(\sqrt{2(x-z)})$, and $2(x-z) \in K(\sqrt{a})$.

On the other hand, suppose that for some $t \in K(\sqrt{a})$, we have $t u \in J^{2}$. Then $\sigma(t)=t$, $(t u) \sigma(t u)=t^{2} u \sigma(u) \in\left(K(\sqrt{a})^{2}\right.$, and so $u \sigma(u) \in\left(K(\sqrt{a})^{2}\right.$. Then $s=0$, and this completes the proof.

Corollary 1.6. Assume the notation above, and fix $i, 1 \leq i \leq 3$. Then the Galois group $\operatorname{Gal}\left(L / K\left(\sqrt{a_{i}}\right)\right)$ is $Z_{2} \times Z_{2}$ if and only if $s_{i}=0$.

Proof. Suppose first that $s_{i}=0$. Then $L=J(\sqrt{t})$ for some $t \in K\left(\sqrt{a_{i}}\right)$, such that $t u \in J^{2}$. Since $u \in J-J^{2}$, then also $t \in J-J^{2}$. Hence $L$ must contain the quartic subfield $F=K\left(\sqrt{a_{i}}, \sqrt{t}\right)$, and $F \neq J$. Then $\operatorname{Gal}\left(L / K\left(\sqrt{a_{i}}\right)\right)$ cannot be $Z_{4}$, and must be $Z_{2} \times Z_{2}$.

On the other hand, suppose that $\operatorname{Gal}\left(L / K\left(\sqrt{a_{i}}\right)\right)$ is $Z_{2} \times Z_{2}$. Then $L$ contains a quartic subfield of the form $F=K\left(\sqrt{a_{i}}, \sqrt{t}\right)$, with $t \in K\left(\sqrt{a_{i}}\right)$, where $F \neq J$. Then for $j \neq i$, we have

$$
K\left(\sqrt{a_{i}}, \sqrt{t}, \sqrt{a_{j}}\right)=L=K\left(\sqrt{a_{i}}, \sqrt{a_{j}}, \sqrt{u}\right),
$$

and it follows that $t u \in J^{2}$. Then $s_{i}=0$.

COROLLARY 1.7. Using the notation above, the fields $L$ for which $\mathrm{Gal}(L / K)$ is one of $Z_{2} \times Z_{2} \times Z_{2}, Z_{4} \times Z_{2}$, or $D_{4}$, are classified and described as follows.

(A) The following are equivalent:

(a) $\operatorname{Gal}(L / K)=Z_{2} \times Z_{2} \times Z_{2}$

(b) $S(u)=(0,0,0)$.

(c) $L=J(\sqrt{b})$ for some $b \in K-J^{2}$.

(B) The following are equivalent:

(a) $\mathrm{Gal}(L / K)=D_{4}$ and $\mathrm{Gal}\left(L / K\left(\sqrt{a_{1}}\right)\right.$ is cyclic

(b) $s_{1}(u)=1$ and $s_{k}(u)=0$ for $k=2,3$,

(c) There exists $x=r+s \sqrt{a_{2}} \in K\left(\sqrt{a_{2}}\right)-J^{2}$ such that $r^{2}-a_{2} s^{2}=a_{3} t^{2}$ for some $t \in K$, and $L=J(\sqrt{x})$.

(C) The following are equivalent:

(a) $\operatorname{Gal}(L / K)=Z_{4} \times Z_{2}$ and $\operatorname{Gal}\left(L / K\left(\sqrt{a_{1}}\right)\right.$ is $Z_{2} \times Z_{2}$,

(b) $s_{1}(u)=0$ and $s_{k}(u)=1$ for $k=2,3$,

(c) There exists $x=r+s \sqrt{a_{1}} \in K\left(\sqrt{a_{1}}\right)-J^{2}$ such that $r^{2}-a_{1} s^{2}=a_{1} t^{2}$ for some $t \in K$, and $L=J(\sqrt{x})$.

(d) There exist $y, z \in K$ such that $a_{1}=y^{2}+z^{2}$.

(D) The following are equivalent:

(a) $\operatorname{Gal}(L / K)=Q_{8}$

(b) $s_{i}(u)=1$ for $i=1,2,3$

(c) If $x=y z$ where $y \in K\left(\sqrt{a_{i}}\right)$ and $z \in K\left(\sqrt{a_{j}}\right)$, then $L \neq J(\sqrt{x})$ 
Proof. Put $N=s_{1}(u)+s_{2}(u)+s_{3}(u)$. Then by Corollary 1.6, the number of quartic subfields of $L$ is $2(3-N)+1$, which is the number of subgroups of order 2 of $\mathrm{Gal}(L / K)$. This number determines a group of order 8 , and so we have: $N=0$ if and only if $\operatorname{Gal}(L / K)$ is $Z_{2} \times Z_{2} \times Z_{2} ; N=1$ if and only if $\operatorname{Gal}(L / K)$ is $D_{4}, N=2$ if and only if $\operatorname{Gal}(L / K)$ is $Z_{4} \times Z_{2}$; and $N=3$ if and only if $\operatorname{Gal}(L / K)$ is $Q_{8}$. (Since $L$ contains $J$, we need not consider $Z_{8}$.)

If $\mathrm{Gal}(L / K)$ is $Z_{2} \times Z_{2} \times Z_{2}$, then $L$ contains quadratic subfields (over $K$ ) other than $K\left(\sqrt{a_{1}}\right)$; if one of these is $K(\sqrt{b})$, then $L=J(\sqrt{b})$, and conversely. This proves (A).

If $\mathrm{Gal}(L / K)$ is $D_{4}$ and $s_{1}(u)=1, s_{2}(u)=s_{3}(u)=0$, and by Corollary 1.6, $\operatorname{Gal}\left(L / K\left(a_{1}\right)\right)$ is $Z_{4}$ and $\operatorname{Gal}\left(L / K\left(\sqrt{a_{i}}\right)\right)$ is $Z_{2} \times Z_{2}$ for $i=2,3$. Then $L$ contains a quadratic extension $M$ of $K\left(\sqrt{a_{2}}\right)$, say, $M=K\left(\sqrt{a_{2}}, \sqrt{x}\right)$ for some $x$ in $K\left(\sqrt{a_{2}}\right)$, and $M$ is not normal over $K$ (since $\operatorname{Gal}(L / K)$ is $\left.D_{4}\right)$. Write $x=r+s \sqrt{a_{2}}$. Then $N_{K}(x)=r^{2}-a_{2} s^{2}$ is not in $M^{2}$; it is in $J^{2}$ however, so we must have that $N_{K}(x)=\alpha t^{2}$, where $\alpha$ is either $a_{1}$ or $a_{3}$. From the identity (ID2), $K(\sqrt{\alpha})$ will have a quadratic extension other than $J$, and so $\alpha$ must be $a_{3}$. The converse is clear, and this proves (B).

If $\operatorname{Gal}(L / K)=Z_{4} \times Z_{2}$ and $\operatorname{Gal}\left(L / K\left(\sqrt{a_{1}}\right)\right.$ is $Z_{2} \times Z_{2}$, then $L$ must contain a normal (over $K$ ) quartic field $M=K\left(\sqrt{a_{1}}, \sqrt{x}\right)$ with $x=r+s \sqrt{a_{1}}$. Since $N_{K}(x) \in M^{2}$, we must have $N_{K}(x)=\alpha t^{2}$, where $\alpha$ is either 1 or $a_{1}$, and $t \in K$. By Corollary 1.2 and (A), if $\alpha=1$, then $\mathrm{Gal}(L / K)$ is $Z_{2} \times Z_{2} \times Z_{2}$, a contradiction. So $\alpha=a_{1}$ as required. The converse is clear. Finally, if we have $x=r+s \sqrt{a_{1}}$ where $r^{2}-a_{1} s^{2}=a_{1} t^{2}$, then none of $r, s, t$ is 0 , and $r^{2}=a_{1}\left(s^{2}+t^{2}\right)$. Take $y=r s /\left(s^{2}+t^{2}\right)$ and $z=r t /\left(s^{2}+t^{2}\right)$; then $a_{1}=y^{2}+z^{2}$, so (d) holds. On the other hand, if we have $a_{1}=y^{2}+z^{2}$, then $a_{1}^{2}-a_{1} y^{2}=a_{1} z^{2}$, and (c) is satisfied. This proves (C).

Now (D) follows from (A), (B), and (C).

2. Quatemionic fields. In this section we discuss some of the relations among statements of the following type (where $G$ and $H$ are non-cyclic groups of order 8 ):

(a) $J$ can be embedded both in a $G$-extension and an $H$-extension

(b) $J$ can be embedded in a $G$-extension and not in an $H$-extension

(c) $J$ can be embedded in neither a $G$-extension nor an $H$-extension.

We shall see that the fields $L$ with $\operatorname{Gal}(L / Q)=Q_{8}$, fall naturally into two distinct classes, or types: A field $L$ with $\operatorname{Gal}(L / K)=Q_{8}$ is of Type $I$ if and only if it has any of the following (equivalent) properties: $J$ has a $D_{4}$-extension; $J$ has a $Z_{2} \times Z_{4}$-extension; $L=J(\sqrt{u})$ where $u=x y$ with $x$ and $y$ elements of two different quadratic subfields of $J$. Otherwise, $L$ is of Type $I I$.

We use all the notation of Section 1.

LEMMA 2.1. Let $u, v, u v \in J-J^{2}$, and assume as always that $L_{1}=J(\sqrt{u}), L_{2}=J(\sqrt{v})$, and $L_{3}=J(\sqrt{u v})$ are normal over $K$. Then we have $S\left(L_{3}\right) \equiv S\left(L_{1}\right)+S\left(L_{2}\right)(\bmod 2)$.

Proof. For each $i, 1 \leq i \leq 3$, we can write

$$
u \sigma_{i}(u)=\alpha r^{2}, \quad v \sigma_{i}(v)=\beta s^{2}, \quad(u v) \sigma_{i}(u v)=(\alpha \beta)(r s)^{2}
$$

for some $r, s \in K\left(\sqrt{a_{i}}\right)$, where $\alpha$ and $\beta$ are either 1 or $a_{j}(j \neq i)$. Clearly, $s_{i}(u v)=0$ if $\alpha=\beta$, and is 1 otherwise. Since $\alpha=\beta$ if and only if $s_{i}(u)=s_{i}(v)$, the result follows. 
Corollary 2.2. Fix $J$, and let $G(J)=G$ be the set of all possible (distinct) ordered triples $S(u)$ for $u \in J-J^{2}$. Then $G$, equipped with pointwise addition modulo two, is a group.

Proof. It was assumed at the beginning that $K$ does have a normal non-cyclic extension of degree 8 (which contains a non-trivial $J$ ) and $\operatorname{char}(K)$ is not 2 . Then $K-J^{2}$ is not empty, for if $x \in J$ and $x^{2} \in K$, then $x=r \sqrt{a_{i}}$ ) for some $i=1,2$, or 3. Take $b \in K-J^{2}$; then by Lemma $1.7 \mathrm{~A}(\mathrm{c}), J(\sqrt{b})$ has $S(b)=(0,0,0)$ and so $(0,0,0)$ is in $G$. By Lemma 2.1 $G$ is closed under its operation, and every element is its own inverse. So $G$ is a group.

It is not difficult to come up with examples (e.g. with $K=Q$ ) to show that all possibilities for $G$ are in fact attained. We give a few examples at the end of this section.

Definition 2.3. If $L_{1}=J(\sqrt{u})$ and $L_{2}=J(\sqrt{v})$ are normal extensions of $K$ such that $S(u)+S(v) \equiv(1,1,1)(\bmod 2)$, then we say that $L_{1}$ and $L_{2}$ are complementary extensions. If $S(u)=S(v)$, then $L_{1}$ and $L_{2}$ are matching extensions.

The next Corollary gives some easy consequences of the fact that $G(J)$ is a group.

Corollary 2.4. Suppose that $J$ has normal (over $K$ ) extensions $L_{1}=J(\sqrt{u}$ ) and $L_{2}=J(\sqrt{v})$.

(a) $L_{1}$ and $L_{2}$ are matching extensions if and only if there exists some element $b \in K$ such that buv $\in J$, that is, $L_{2}=J(\sqrt{b u})$.

(b) $J$ has a $Q_{8}$ extension if and only if $J$ has at least one pair of complementary extensions.

(c) If $J$ has a $Q_{8}$ extension, then $J$ has a $D_{4}$ extension if and only if $J$ has a $Z_{2} \times Z_{4}$ extension.

(d) If $J$ has three $D_{4}$ extensions which are pairwise not matching, then $G(J)$ has order 8 , and $J$ has extensions of every kind.

The next result characterizes $Q_{8}$ extensions of Type $I$ according to the properties of the defining element $u$.

THEOREM 2.5. Suppose that $L=J(\sqrt{u})$ with $\operatorname{Gal}(L / Q)=Q_{8}$. Then the following are equivalent:

(a) $J$ has a $D_{4}$ extension

(b) J has a $Z_{2} \times Z_{4}$ extension

(c) For some $i \neq j, 1 \leq i, j \leq 3$, there exist elements $x \in K\left(\sqrt{a_{i}}\right)-K$ and $y \in K\left(\sqrt{a_{j}}\right)-$ $K$ such that $L=J(\sqrt{x y})$.

Proof. The equivalence of (a) and (b) is given in Corollary 2.4. Assume (a); suppose that $J$ has a $D_{4}$ extension $M$ cyclic over $K\left(\sqrt{a_{1}}\right)$. By Corollary $1.7, L$ has a quartic subfield $M=J(\sqrt{x})$ where $x \in K\left(\sqrt{a_{2}}\right)-K$. We have $S(u)=(1,1,1)$ by assumption, and $S(x)=$ $(1,0,0)$. Then $S(u x)=(0,1,1)$, so that $N=J(\sqrt{u x})$ has $\operatorname{Gal}(N / K)=Z_{2} \times Z_{4}$. Again by Corollary 1.7 , there is an element $y \in K\left(\sqrt{a_{1}}\right)-K$ so that $N=J(\sqrt{y})$. Now it follows that $u x y \in J^{2}$, that is, $L=J(\sqrt{u})=J(\sqrt{x y})$.

Now assume (c); suppose that $x \in K\left(\sqrt{a_{1}}\right)-K$ and $y \in K\left(\sqrt{a_{2}}\right)-K$. Since $L$ is normal and $\operatorname{Gal}(L / K)=Q_{8}$, then neither $x$ nor $y$ is in $J^{2}$, and we have $(x y) \sigma_{1}(x y)=$ $x^{2} y \sigma_{1}(y) \in a_{2}\left(K\left(\sqrt{a_{1}}\right)\right)^{2}$. Since $y \sigma_{1}(y) \in K$, we must have either $y \sigma_{1}(y) \in a_{2} K^{2}$ or $y \sigma_{1}(y) \in$ 
$\mathrm{a}_{1} \mathrm{a}_{2} \mathrm{~K}^{2}$. Then $J(\sqrt{y})$ is a normal field, and its Galois group is either $D_{4}$ (and (a) holds) or $Z_{2} \times Z_{4}$ (and (b) holds). This completes the proof.

The argument of Theorem 2.5 indicates a method of construction for a $Q_{8}$ extension of Type $\mathrm{I}$, that is, one need only find appropriate $x$ and $y$ in quadratic subfields of $J$, satisfying (respectively) conditions $B(c)$ and $C(c)$ of Corollary 1.7 .

If $K$ has a $D_{4}$ extension $L_{1}$ and a $Z_{2} \times Z_{4}$ extension $L_{2}$, which are complementary, then the composite field $M=L_{1} L_{2}$ is normal over $K$ and has degree 16 over $K$. Thus we have the following corollary.

COROLlary 2.6. $J$ has a $Q_{8}$ extension of Type $I$ if and only if $J$ can be embedded into a normal extension $M$ of $K$ where $\mathrm{Gal}(M / K)$ is the group of order 16 generated by two elements $\rho$ and $\tau$ satisfying the relations

$$
\rho^{4}=\tau^{4}=\rho \tau \rho \tau^{-1}=1 .
$$

Proof. In [6], Thomas and Wood give the subgroup lattices for all groups of order 16. Since $M$ is a composite field, $M=L_{1} L_{2}$, it is easy to count up the subfields of $M$ according to their degree over $K$ : there are three of degree 2 , seven of degree 4 , and three of degree 8 . Since $\operatorname{Gal}(M / K)$ is not abelian, there is only one possibility; it has generators as described.

We now give some examples to illustrate the possibilities. We use some of the work of the next section, on quaternionic extensions of $Q$.

Example 2.7. A $Q_{8}$ extension of Type I. Let $K=Q, J=Q(\sqrt{2}, \sqrt{3})$. Take $u=2+\sqrt{2}$, and $v=3+\sqrt{3}$. Then $L_{1}=J(\sqrt{u})$ has $\operatorname{Gal}\left(L_{1}\right)=Z_{2} \times Z_{4}$, and $L_{2}=J(\sqrt{v})$ has $\operatorname{Gal}\left(L_{2}\right)=D_{4}$; furthermore, $L_{1}$ and $L_{2}$ are complementary. Then $J(\sqrt{u v})$ is quaternionic, and of Type $I$.

EXAmple 2.8. A $Q_{8}$ extension of Type II. Let $K=Q, J=Q(\sqrt{3}, \sqrt{14})$. Take $x=5+3 \sqrt{3}, y=7+2 \sqrt{14}$, and $z=6+\sqrt{42}$. Then put $u=x y z$, and $L=J(\sqrt{u})$. We have $u \sigma_{1}(u)=42 x^{2}, u \sigma_{2}(u)=12 y^{2}$ and $u \sigma_{3}(u)=14 z^{2}$, so that $\operatorname{Gal}(L)=Q_{8}$. Since none of 3 , 14,42 is a sum of two integer squares, $J$ has no $Z_{2} \times Z_{4}$ extensions, and hence no $D_{4}$ extensions.

Example 2.9. A field with a $D_{4}$ extension, but no $Q_{8}$ extension. Let $K=Q$, and $J=Q(\sqrt{2}, \sqrt{7})$. Then if $x=3+\sqrt{2}, L_{1}=J(\sqrt{x})$ is dihedral. By a result of Reichardt [4], since $7 \equiv 7(\bmod 8), J$ has no $Q_{8}$ extension.

3. Type II extensions. In this section, $K$ is the rational field $Q$. The results hold for $Q_{8}$ extensions of both types, but the primary interest is in their application as a method for constructing $Q_{8}$ extensions of Type II. We assume that $a_{1}=m, a_{2}=n$, and $a_{3}=k=m n / \operatorname{gcd}(m, n)^{2}$ are positive squarefree integers, with $m n$ not square. (Recall that a $Q_{8}$ extension of $Q$ is a real field.) Otherwise, we use the notation of Section 1 .

LEMMA 3.1. Suppose that $u=x_{1} x_{2} x_{3}$, where $x_{i} \in K\left(\sqrt{a_{i}}\right)$, for $i=1,2,3$, and that $L=J(\sqrt{u})$ is normal over $Q$. Assume that each $x_{i}$ is an algebraic integer, and put $r_{1}=N\left(x_{2}\right) N\left(x_{3}\right), r_{2}=N\left(x_{1}\right) N\left(x_{3}\right)$, and $r_{3}=N\left(x_{1}\right) N\left(x_{2}\right)$. Then each $r_{i}$ is a rational integer, and we write $r_{i}=m_{i} t_{i}^{2}$, where $m_{i}$ and $t_{i}$ are integers and $m_{i}$ is squarefree. (a) For $i=1,2,3$, $m_{i} \in\{1, m, n, k\}$. (b) If any two of $m_{1}, m_{2}, m_{3}$ are equal, then $\mathrm{Gal}(L / Q)$ is not $Q_{8}$. 
Proof. Since $L$ is normal over $Q$, then $u \sigma_{i}(u)=r_{i} x_{i}^{2}$ is square in $J$ for each $i=1,2,3$, so that $r_{i}$ is square in $J$. Then there is some $a \in\{1, m, n, k\}$, such that $a r_{i}$ is a rational square. Since $a$ is squarefree and $r_{i}$ is an integer, (a) follows. To see (b), suppose e.g. that $m_{1}=m_{2}$. Then $r_{1} r_{2}=r_{3} N\left(x_{3}\right)^{2}$ is a rational square, and then $r_{3}$ is a rational square, and $u \sigma_{3}(u)=r_{3} x_{3}^{2}$ is square in $J$. This proves (b).

Corollary 3.2. If $\operatorname{Gal}(L / Q)=Q_{8}$, then $\left(m_{1}, m_{2}, m_{3}\right)$ is either $\left(a_{2}, a_{3}, a_{1}\right)$ or $\left(a_{3}, a_{1}, a_{2}\right)$.

Remark. At this point, it is easy to see that if $K$ has a $Q_{8}$ extension $L$ as in Lemma 3.1 , then $K$ also has a $D_{4}$ extension: one of the fields $K\left(\sqrt{a_{i}}, \sqrt{x_{i}}\right)$ must have $D_{4}$ as its normal closure. Stronger results of this nature are proved in $[8]$.

We are interested in constructing $Q_{8}$ extensions of $J=Q(\sqrt{m}, \sqrt{n})$ using properties of the integers in the quadratic subfields of $J$. Obviously, some of our results could be generalized directly to any field $K$ with a sufficiently well-behaved ring of integers.

THEOREM 3.3. Let $K=Q$, and suppose $a_{1}=m, a_{2}=n$ are squarefree integers such that $m n$ is not square. Define $D=\operatorname{gcd}(m, n)$, and write $m=M D, n=N D$. Put $a_{3}=k=M N$, and $J=Q(\sqrt{m}, \sqrt{n})$. Then $J$ has a $Q_{8}$ extension $L$ if and only if there exist rational integers $j, m_{1}, m_{2}, n_{1}, n_{2}, d_{1}, d_{2}$, and integers $x \in Q(\sqrt{m}), y \in Q(\sqrt{n}), z \in Q(\sqrt{k})$, such that

(1) $M=m_{1} m_{2}, N=n_{1} n_{2}, D=d_{1} d_{2}$

(2) $N(x)=n_{1} m_{1} d_{1} j t^{2}$

(3) $N(y)=n_{2} m_{1} d_{2} j r^{2}$

(4) $N(z)=n_{1} m_{2} d_{2} j s^{2}$

for some $r, s, t \in Q$.

Proof. Suppose first that all the conditions above are satisfied, and put $u=x y z$. Then $u \sigma_{1}(u)=x^{2} N(y) N(z)=N M\left(x d_{2} j r s\right)^{2}=k \alpha^{2}$ with $\alpha \in Q(\sqrt{m})$; similarly $u \sigma_{2}(u)=$ $m \beta^{2}$ for some $\beta \in Q(\sqrt{n})$ and $u \sigma_{3}(u)=n \gamma^{2}$ for some $\gamma \in Q(\sqrt{k})$. Then $S(u)=(1,1,1)$ and $\operatorname{Gal}(J(\sqrt{u}))$ is $Q_{8}$.

Conversely, suppose that $S(u)=(1,1,1)$. By Lemma 1.3 , and multiplying by a rational integer square if necessary, we may assume that $u=x y z$, where $x \in Q(\sqrt{m})$, $y \in Q(\sqrt{n})$, and $z \in Q(\sqrt{k})$, are algebraic integers. Then the norms $N(x), N(y), N(z)$ are rational integers, and by Corollary 3.2, the integers $k N(y) N(z), m N(x) N(z)$, and $n N(x) N(y)$ are rational squares. The argument is based on the fact that $M, N$, and $D$ are squarefree, positive, and pairwise relatively prime.

If $k N(y) N(z)$ is square, then (since $k=M N$ ) there are integers so that $N(y)=n_{2} m_{1} a$ and $N(z)=n_{1} m_{2} b$ with $n_{1} n_{2}=N$ and $m_{1} m_{2}=M$. Then if $m N(x) N(z)$ is square, there must be integers so that $N(x)=m_{1} d_{1} c$ and $N(z)=m_{2} d_{2} d$, where $d_{1} d_{2}=D$. It follows that $N(z)=n_{1} m_{2} d_{2} \alpha$ for some rational integer $\alpha$. Similarly, $N(y)=n_{2} m_{1} d_{2} \beta$ and $N(x)=$ $n_{1} m_{1} d_{1} \gamma$, for rational integers $\beta$ and $\gamma$. Now $k N(y) N(z)=k N M d_{2}^{2} \alpha \beta=\left(k d_{2}\right)^{2} \alpha \beta$ is a rational square, and so $\alpha$ and $\beta$ must have the same squarefree part. The same is true for $\alpha$ and $\gamma$, and for $\beta$ and $\gamma$. Put $j$ equal to this common squarefree part, and conditions (1)-(4) are satisfied.

Notation. If $p$ is an odd prime and $a$ is an integer, then we use the notation $(a \mid p)$ for the Legendre symbol: if $(a, p)=1$, then $(a \mid p)=1$ is a square $\bmod p$, and is -1 otherwise. 
General residuacity conditions for the existence of a $Q_{8}$ extension of $J$ are known; see e.g. [2] or [4]. The next theorem gives detailed conditions involving the integers $n_{i}$, $m_{i}, d_{i}$ of Theorem 3.3.

THEOREM 3.4. We use the notation of Theorem 3.3. Let $J(\sqrt{x y z})$ be a $Q_{8}$ extension of $J$, such that conditions (1)-(4) of Theorem 3.3 are satsified. Let $p$ be an odd prime.

(a) If $p \mid m_{1} n_{1} d_{2}$, then $p \equiv 1(\bmod 4)$.

(b) If $p \mid N$, then $(-m \mid p)=1$.

(c) If $p \mid M$, then $(-n \mid p)=1$.

(d) If $p \mid D$ then $(-k \mid p)=1$.

Proof. Let $p$ be an odd prime, and write $x=a+b \sqrt{m}, y=c+d \sqrt{n}, z=e+f \sqrt{k}$, where $a, b, c, d, e, f$ are rational numbers with denominator 1 or 2 . Since $p$ is an odd prime, we may as well assume that they are all integers; we can also assume that each of $\operatorname{gcd}(a, b), \operatorname{gcd}(c, d)$, and $\operatorname{gcd}(e, f)$ is 1 or 2 . Then

(i) $N(x)=a^{2}-m b^{2}=n_{1} m_{1} d_{1} j t^{2}$

(ii) $N(y)=c^{2}-n d^{2}=n_{2} m_{1} d_{2} j r^{2}$

(iii) $N(z)=e^{2}-k f^{2}=n_{1} m_{2} d_{2} j s^{2}$.

(a) Suppose first that $p\left(m_{1}\right)$. Then $p \mid m$, and from (i), $p \mid a$, so equation (i) is divisible by $p$. We also have $p \mid k$, and so equations (i) and (iii) yield the congruences

$$
\begin{aligned}
-(m / p) b^{2} & \equiv n_{1}\left(m_{1} / p\right) d_{1} j t^{2} \quad(\bmod p), \\
e^{2} & \equiv n_{1} m_{2} d_{2} j s^{2} \quad(\bmod p) .
\end{aligned}
$$

Since $m=m_{1} m_{2} d_{1} d_{2}$, then multiplying these together gives

$$
-(m / p) b^{2} e^{2} \equiv n_{1}^{2}(m / p) j^{2} t^{2} s^{2} \quad(\bmod p) .
$$

Since $\operatorname{gcd}(a, b)$ and $\operatorname{gcd}(e, f)$ can only be 1 or 2 , and $p$ is odd, then $\operatorname{gcd}(b, p)=1$ and $\operatorname{gcd}(e, p)=1$. Of course $\operatorname{gcd}((m / p), p)=1$, and so $(-1 \mid p)=1$, and $p \equiv 1(\bmod 4)$. The proof is similar if $p \mid n_{1}$ or $p \mid d_{2}$.

The statements (b), (c), (d) are all proved in the same way; we give the argument for (c). If $p \mid m_{1}$, then $(n \mid p)=1$ from equation (ii). Since $(-1 \mid p)=1$ also, then $(-n \mid p)=$ 1. Suppose now that $p \mid m_{2}$. Since $k=M N$, then $m_{2} \mid k$, and then $p \mid e$, so equation (iii) is divisible by $p$. Then equations (i) and (iii) give the congruences

$$
\begin{aligned}
-(k / p) f^{2} & \equiv n_{1}\left(m_{2} / p\right) d_{2} j s^{2} \quad(\bmod p) . \\
a^{2} & \equiv n_{1} m_{1} d_{1} j t^{2} \quad(\bmod p) .
\end{aligned}
$$

Multiplying these together gives

$$
-(k / p) f^{2} a^{2} \equiv n_{1}^{2}(M / p) D j^{2} s^{2} t^{2} \quad(\bmod p) .
$$

and since $(k / p)=N(M / P)$ and $n=N D$, it follows that $(-n \mid p)=1$.

The next Corollary is helpful in finding solutions to the equations (1)-(4) of Theorem 3.3 .

Corollary 3.5. Suppose that $J$ has a $Q_{8}$ extension, and assume the notation of Theorem 3.3. Suppose that $p$ is an odd prime divisor of $m n$. Then $p$ is inert in one of the 
quadratic subfields of $J$ if and only if $p \equiv 3(\bmod 4)$, and $p$ splits in one of the quadratic subfields of $J$ if and only if $p \equiv 1(\bmod 4)$.

Proof. It is well known that a prime $p$ splits (resp. is inert) in a quadratic field $Q(\sqrt{j})$ if and only if $\operatorname{gcd}(j, p)=1$, and $(j \mid p)=1 \quad(\operatorname{resp} .(j \mid p)=-1)$. The result follows immediately from Theorem 3.4 .

4. Applications. We work out some specific constructions for $n, m$ such that $J=Q(\sqrt{m}, \sqrt{n})$ has a $Q_{8}$ extension. As before, $m$ and $n$ are positive squarefree integers such that $m n$ is not square. We use, without much comment, various special properties of quadratic fields; for instance if $x \in Q(\sqrt{j})$ and $j<0$, then $N(x) \geq 0$; the multiplicative properties of norms, and so on.

The residuacity conditions for odd primes are easily computed from Theorem 3.4 or otherwise. Congruence conditions on $(m, n, k)$ modulo 8 are given by Reichardt in [4]: we must have either (a) $m \equiv n \equiv 1(\bmod 4)$, or else (b) $m \equiv n \equiv 2 \bmod 4)$, and $(m / 2, n / 2, k)$ congruent to one of $(1,1,1),(3,7,5),(5,5,1),(1,3,3),(5,7,3)(\bmod 8)$.

In the examples below, one of $n, m$ is a prime. The following lemma gives some useful consequences of Lemma 1.1 for rational integers.

Lemma 4.1. Let $p$ be an odd prime, and let $n= \pm p$. Suppose that $x, y$ are integers such that $\operatorname{gcd}(x, y)=1$. (a) If $t$ is odd and $x^{2}-n y^{2}=t^{2}$, then one of the quantities $\pm(x+y \sqrt{n})$ is a square in $Q(\sqrt{n})$. (b) If $t$ is odd and $x^{2}-n y^{2}=4 t^{2}$, then there exist integers $\alpha, \beta$ such that $\alpha^{2} \pm p \beta^{2}= \pm 4 t$.

Proof. (a) From the assumptions, $(x-t) / 2$ and $(x+t) / 2$ are relatively prime integers, and their product is $\pm p(y / 2)^{2}$. Then since $p$ is prime, we have, say, $(x-t) / 2= \pm p \alpha^{2}$ and $(x+t) / 2= \pm \beta^{2}$, for integers $\alpha$ and $\beta$. Then by the identity (ID1) of Lemma 1.1, one of $\pm(x+y \sqrt{n})$ is square. Similarly, for (b), one of the quantities $\pm(x-2 t)$ is square in $Q(\sqrt{n})$. That is, one of $\pm 2(x+y \sqrt{n})$ is equal to a square, say, $(\alpha+\beta \sqrt{n})^{2}$. Then $\alpha^{2}-n \beta^{2}= \pm 4 t$.

ExAmple 4.2. $J=Q(\sqrt{2}, \sqrt{n})$ has a $Q_{8}$ extension if and only if every odd prime factor $p$ of $n$ satisfies $p \equiv 1$ or $3(\bmod 8)$.

Since $2=1^{2}+1^{2}$, a $Q_{8}$ extension of $J$ must be of Type I. Suppose that $n$ is odd. Let $y=2+\sqrt{2}$; then $y$ satisfies condition $\mathrm{C}(\mathrm{c})$ of Corollary 1.7 . It remains to find an $x \in Q(\sqrt{2 n})$ satisfying condition $\mathrm{B}(\mathrm{c})$ of Corollary 1.7 , that is,

$$
x=a+b \sqrt{2 n} \text { and } N(x)=a^{2}-2 n b^{2}=n t^{2}
$$

(where we may assume $a, b$ and $t$ are rational integers). If $a^{2}-2 n b^{2}=n t^{2}$, then (since $n$ is squarefree) $n \mid a$, say $a=n c$. Then $n^{2} c^{2}-2 n b^{2}=n t^{2}$, and $n c^{2}-2 b^{2}=t^{2}$, from which we have $n c^{2}=t^{2}+2 b^{2}$. Since $Q(\sqrt{-2})$ is a PID, this equation is solvable if and only if $n \equiv 1$ or $3(\bmod 8)$; in this case, there is a solution $x$ for $(1)$, and then $J(\sqrt{x y})$ is a $Q_{8}$ extension.

EXAmple 4.3. Let $p \equiv q \equiv 1(\bmod 4)$ be primes. Then $J=Q(\sqrt{p}, \sqrt{q})$ has a $Q_{8}$ extension if and only if $(p \mid q)=1$.

Any $Q_{8}$ extension must be of Type I. We need a solution to $x^{2}-p y^{2}=q t^{2}$. Since $Q(\sqrt{p})$ has odd class number (see e.g. [1]), say $h=2 r+1$, we can always solve either 
$x^{2}-p y^{2}=q^{h}$ or $x^{2}-p y^{2}=4 q^{h}$ (in integers $x, y$ ). Then we can take $t=q^{r}$ or $2 q^{r}$, accordingly, and let $X=x+y \sqrt{p}$. Next, there are integers $u, v$ so that $p q=u^{2}+v^{2}$. Put $Y=p q+u \sqrt{p q}$. Then $J(\sqrt{X Y})$ is a $Q_{8}$ extension.

ExAmple 4.4. Let $p \equiv 1(\bmod 4)$ and $q \equiv 3(\bmod 4)$. Then $J=Q(\sqrt{p}, \sqrt{2 q})$ has a $Q_{8}$ if and only if $p \equiv 5(\bmod 8)$ and $(p \mid q)=-1$.

Any $Q_{8}$ extension must be of Type $I$. We need a solution to the equation $x^{2}-2 q y^{2}=2 p q t^{2}$, or equivalently, a solution to $a^{2}+p b^{2}=2 q c^{2}$.

In $Q(\sqrt{-p})$, the class group has even order, and we have the prime ideal factorizations: (2) $=P^{2}$ and $(q)=Q_{1} Q_{2}$. Suppose that $Q_{1}$ were of odd order $t$ in the class group. Then there exist integers $x, y$ such that $x^{2}+p y^{2}=q^{t} ; x$ and $y$ must be of opposite parity since $q^{t}$ is odd. But then $x^{2}+p y^{2} \equiv 1(\bmod 4)$, while $q^{t} \equiv 3(\bmod 4)$, a contradiction. Thus $Q_{1}$ has even order, say $2 j$, in the class group.

Now there must be integers $x, y$ so that $x^{2}+p y^{2}=q^{2 j}$. From Lemma 4.1, if $x$ is odd, then one of $\pm(x+y \sqrt{-p})$ is square, and $2 j$ is not minimal. So $x$ must be even. We next show that $j$ is odd. Since $\left(x-q^{j}\right)\left(x+q^{j}\right)=-p y^{2}$, we may suppose that $x-q^{j}= \pm s^{2}$ and $x+q^{j}= \pm p r^{2}$, with $r s=y$. Then (with appropriate choice of sign)

$$
\pm 2(x+y \sqrt{-p})=[s+(y / s) \sqrt{-p}]^{2}=Y^{2}
$$

where $N(Y)=2 q^{j}$. Since $p=5(\bmod 8)$, the equation $u^{2}+p v^{2}=2 z^{2}$ has no integral solutions, so $N(Y)=2 q^{j}$ implies that $j$ must be odd, say $j=2 i+1$. Now we can write $N(Y)=2 q\left(q^{i}\right)^{2}$, as required. Write $Y=a+b \sqrt{-p}$ and $N(Y)=2 q c^{2}$, and let $Z=2 q c-$ $a \sqrt{2 q}$. Then $N(Z)=2 p q b^{2}$. Finally, write $p=r^{2}+s^{2}$, and let $X=p+r \sqrt{p}$. Then $J(\sqrt{X Z})$ is a $Q_{8}$ extension.

The construction of a Type I extension is probably most easily accomplished by the method of the examples above. We do not have anything this simple for Type II extensions, but the next theorem gives a construction for a large class of Type II extensions. This theorem can also be used for Type I extensions.

THEOREM 4.5. Let $\operatorname{gcd}(m, n)=1$, and $J=Q(\sqrt{m}, \sqrt{n})$. Suppose that there are integers such that
(i) $n=a k$
(ii) $a=A^{2}+B^{2}$
(iii) $(A r)^{2}+m s^{2}=k t^{2}$
(iv) $u^{2}+a v^{2}=m w^{2}$.

Then define algebraic integers $X, Y$, and $Z$ by

$$
\begin{aligned}
& X=(B r+s \sqrt{m})(u+w \sqrt{m})=(B r u+m s w)+(u s+B r w) \sqrt{m} \\
& Y=k t^{2}+t r \sqrt{n} \\
& Z=a m s+t B \sqrt{n m}
\end{aligned}
$$

Then $J(\sqrt{X Y Z})$ is a $Q_{8}$ extension of $J$.

Proof. We will show that conditions (1)-(4) of Theorem 3.3 are satisfied. We have $M=m, N=n, D=1$, and we take $m_{1}=1, m_{2}=m, n_{1}=a$ and $n_{2}=k$. Since $D=1$, $d_{1}=d_{2}=1$ can be ignored. Define the integer $j=k t^{2}-a r^{2}$. Then $-j=(B r)^{2}-m s^{2}$, and 
from (iv), we have $u^{2}-m w^{2}=-a v^{2}$. Then since $n=a k$,

$$
\begin{aligned}
& N(X)=\left((B r)^{2}-m s^{2}\right)\left(u^{2}-m w^{2}\right)=a j v^{2}=n_{1} m_{1} j v^{2} \\
& N(Y)=\left(k t^{2}\right)^{2}-n t^{2} r^{2}=k t^{2}\left(k t^{2}-a r^{2}\right)=k j t^{2}=n_{2} m_{1} j t^{2} \\
& N(Z)=(a m s)^{2}-n m(t B)^{2}=a m\left(a m s^{2}-k t^{2} B^{2}\right)=a m j A^{2}=n_{1} m_{2} j A^{2}
\end{aligned}
$$

and the conditions of Theorem 3.3 are satisfied. Then $J(\sqrt{X Y Z})$ is a $Q_{8}$ extension.

ExAmple 4.6. $J=Q(\sqrt{3}, \sqrt{n})$ has a $Q_{8}$ extension if and only if $n=2(\bmod 4)$ and every odd prime divisor $p$ of $n$ satisfies $p \equiv 1$ or $7(\bmod 12)$. The necessity of these conditions follows from the general residuacity conditions given by Reichardt. To see the sufficiency, write $n=2 k$. Since $Q(\sqrt{-3})$ is a PID, and every prime divisor of $k$ splits in $Q(\sqrt{-3})$, then there exist integers $r, s$ so that $r^{2}+3 s^{2}=k$. Then in the notation of Theorem 4.5, we take: $m=3, n=2 k, a=2, A=B=1, t=1, u=v=w=1$, and $r, s$ so that $r^{2}+3 s^{2}=k$. The corresponding $X, Y, Z$ produce the desired $Q_{8}$ extension.

Similar results are possible for $J=Q(\sqrt{p}, \sqrt{2 k})$, where $p$ is a prime, $p=3(\bmod 8)$, as in the next example.

EXAMPLE 4.7. Let $p \equiv 3(\bmod 8)$ and $q \equiv 3(\bmod 4)$. Then $J=Q(\sqrt{p}, \sqrt{2 q})$ has a $Q_{8}$ extension if and only if $(p \mid q)=-1$. Again we need only establish sufficiency. We use Theorem 4.5 with $m=p, n=2 q, m_{1}=1, m_{2}=p, n_{1}=2, n_{2}=q, D=d_{1}=d_{2}=1$. Take $a=2, A=B=1$, and $k=q$. Since $Q(\sqrt{-2})$ is a PID, and since $p=3(\bmod 8)$, there are integers $u, v$ so that $u^{2}+2 v^{2}=p$. We need only show that $r^{2}+p s^{2}=q t^{2}$ has a solution. Since $(p \mid q)=-1$ and $q \equiv 3(\bmod 4)$, then $(-p \mid q)=1$, and $q$ splits in $Q(\sqrt{-p})$. Then there is some minimal integer $j$ so that, for some $x, y \in Z$, either

$$
\text { (a) } x^{2}+p y^{2}=q^{j} \text { or (b) } x^{2}+p y^{2}=4 q^{j} \text {. }
$$

If (a) holds, then $x$ and $y$ have opposite parity. Suppose that $j$ is even, $j=2 i$. Then $q^{j} \equiv 1(\bmod 4)$, and since $p \equiv 3(\bmod 8)$, we must have $x$ odd and $y \equiv 0(\bmod 4)$. Then by Lemma 4.1 , one of $\pm(x+y \sqrt{-p})$ is square, and its square roots has norm $q^{i}$; then $j$ is not minimal. Thus in case (a), $j$ must be odd.

If (b) holds, then $x$ and $y$ are odd. If $j$ is even, $j=2 i$, then by Lemma 4.1 , there would be an element of norm $4 q^{i}$ and $j$ would not be minimal. So in case (b) also, $j$ must be odd.

It follows that the equation $r^{2}+p s^{2}=q t^{2}$ has an integral solution. Then the conditions of Theorem 4.5 are satisfied, and the corresponding $X, Y$, and $Z$ give a $Q_{8}$ extension $J(\sqrt{X Y Z})$.

4.8. Let $m \equiv 3(\bmod 8), n \equiv 2(\bmod 4)$, with $\operatorname{gcd}(m, n)=1$, and suppose that both $Q(\sqrt{m})$ and $Q(\sqrt{-m})$ are PID's. If all the residuacity conditions are satisfied, then $J=Q(\sqrt{m}, \sqrt{n})$ has a $Q_{8}$ extension.

Proof. We use Theorem 4.5. Write $n=a k$, where $k$ is the product of all the prime factors of $n$ which are congruent to $3(\bmod 4)$, and $a$ is the product of the remaining factors. Then $a=A^{2}+B^{2}$ for some integers $A, B$, and $a \equiv 2(\bmod 8)$. If $p \mid k$, then $(-m \mid p)=1$, and so since $Q(\sqrt{-m})$ is a PID, the equation $x^{2}+m y^{2}=k$ has an integral solution. Multiplying through by $A^{2}$, we have a solution to the equation $(A r)^{2}+m s^{2}=k t^{2}$. 
If $p \mid a$ and $p$ is odd, then $p \equiv 1(\bmod 4)$, so $(-m \mid p)=1$ implies $(m \mid p)=1$. Since $m \equiv 3(\bmod 8), 2$ ramifies in $Q(\sqrt{m})$. Now since $Q(\sqrt{m})$ is a PID, there must be a solution to either $x^{2}-m y^{2}=a$, or to $x^{2}-m y^{2}=-a$. Since $m=3(\bmod 8)$ and $a \equiv 2(\bmod 8)$, the equation $x^{2}-m y^{2}=a$ is not possible. Then there is a solution to $x^{2}-m y^{2}=-a$, and then $x^{2}+a=m y^{2}$. Now take $m_{1}=1, m_{2}=m, n_{1}=a$, and $n_{2}=k$, and we have the solution given in Theorem 4.5 .

\section{REFERENCES}

1. Ezra Brown, Class numbers of real quadratic fields, Trans. Amer. Math. Soc. 190 (1974), 99-107.

2. G. Bucht, Uber einige algebraische Korper achten Grades, Arkiv for Matematik, Astronomi och Fysik, Bd. 6, 30 (1910), 1-36.

3. Harvey Cohn, Quaternionic compositum genus, J. Number Theory, 11, (1979), 399-411.

4. Christian U. Jensen and Noriko Yui, Quaternion Extensions, Algebraic Geometry and Commutative Algebra (1987), 155-182.

5. H. Reichardt, Uber Normalkorper mit Qaternionengruppe, Math. Zeitschrift 41, (1936), 218-221.

6. Emanuel Rosenbluth, Die Arithmetische Theorie und die Konstruktion der Quaternionenkorper auf klassenkorpertheoretischer Grundlage, Monatshefte fur Math. u. Phys. 41 (1934), $85-125$.

7. A. D. Thomas and G. V. Wood, Group tables (Shiva Publishing, 1980).

8. Roger Ware, A note on the quaternion group as Galois group, Proc. Amer. Math. Soc. 108, (1990), 621-625.

9. E. Witt, Konstruktion von galoisschen Korpern der Charakteristik p zu vorgegebener Gruppe der Ordnung $p^{f}$, J. Reine Angew. Math. 174 (1936), 237-245.

University of North Carolina at Greensboro, GREENSBORO,

NC 27412,

\section{USA}

